\title{
COBERTURA Y FACTORES ASOCIADOS A LA VACUNACIÓN CONTRA EL SARAMPIÓN EN NIÑOS DE 12 A 59 MESES EN PERÚ: ESTIMACIÓN BASADA EN LA ENCUESTA DEMOGRÁFICA Y DE SALUD FAMILIAR 2017
}

\author{
Karina Vásquez-Uriarte ${ }^{1, a}$, Juana Aurelia Ninatanta Ortiz ${ }^{1, b}$, Franco Romani ${ }^{2, c}$, \\ Joel Christian Roque-Henriquez ${ }^{2,3, d}$
}

RESUMEN

Objetivos. Estimar la cobertura y determinar los factores asociados a la vacunación contra el sarampión en Perú. Materiales y métodos. Realizamos un estudio de fuente secundaria utilizando la Encuesta Demográfica y de Salud Familiar (ENDES) del 2017, la unidad informante fue una mujer en edad fértil de 15 a 49 años; la unidad de análisis fue un niño de 12 a 59 meses (para la primera dosis) o niño de 18 a 59 meses (para la dosis de refuerzo) y que contaba con datos de vacunación. Los datos de cobertura fueron obtenidos de la tarjeta de vacunación. Resultados. Según la tarjeta de vacunación, la cobertura para la primera dosis fue del 70,2\% (IC95\%: 68,8-71,6), para la dosis de refuerzo del $52,0 \%$ (IC95\%: 50,5-53,6). Los niños de 24-35 meses tuvieron más probabilidades de ser vacunados para la primera dosis (OR: 1,59; IC95\%: 1,28-1,97) y dosis de refuerzo (OR:2,04; IC95\%: 1,62-2,56) comparado con los niños de 12-23 meses y 18-23 meses respectivamente. Los niños cuyo control de crecimiento y desarrollo fue en el sector privado tuvieron menores probabilidades de ser vacunados para la primera dosis (OR: 0,30; IC95\%: 0,21-0,43) y dosis de refuerzo (OR: 0,26; IC95\%: 0,17-0,40) comparado con los que se controlaron en el sector público. Conclusiones. Según la ENDES 2017, Perú y ninguna de sus regiones alcanzó una cobertura del 95,0\% para la primera dosis y su refuerzo. El control de crecimiento y desarrollo en establecimientos del sector público está asociado con la vacunación de sarampión en su primera dosis y refuerzo.

Palabras clave: Sarampión; Vacunación; Cobertura de vacunación; Perú (fuente: DeCS BIREME).

\section{COVERAGE AND FACTORS ASSOCIATED WITH MEASLES VACCINATION IN CHILDREN AGED 12-59 MONTHS IN PERU: ESTIMATE BASED ON THE 2017 DEMOGRAPHIC AND FAMILY HEALTH SURVEY}

\begin{abstract}
Objectives. To estimate coverage and determine factors associated with measles vaccination in Peru. Materials and Methods. We conducted a secondary source study using the 2017 Demographic and Family Health Survey (ENDES). The reporting unit was a woman of childbearing age, 15 to 49 years; the unit of analysis was a child, 12 to 59 months (for the first dose), or a child, 18 to 59 months (for the booster dose) who had vaccination information. Coverage data were obtained from the vaccination card. Results. According to the vaccination card, coverage for the first dose was $70.2 \%$ (95\% Cl: $68.8-71.6)$; for the booster dose, $52 \%(95 \% \mathrm{Cl}: 50.5-53.6)$. Children aged $24-35$ months were more likely to be vaccinated for the first dose (OR 1.59, 95\% Cl: 1.28-1.97) and booster dose (OR 2.04, 95\% Cl: 1.62-2.56), compared with children aged 12-23 months and 18-23 months respectively. Children with growth and development check-ups performed in the private sector were less likely to be vaccinated for the first dose (OR $0.30,95 \% \mathrm{Cl}: 0.21-0.43$ ) and booster dose (OR $0.26,95 \% \mathrm{Cl}: 0.17-0.40$ ), compared to those being monitored in the public sector. Conclusions. According to ENDES 2017, Peru and none of its regions achieved 95.0\% coverage for the first and booster doses. Growth and development monitoring in public sector facilities is associated with measles vaccination in terms of first and booster doses.
\end{abstract}

Keywords: Measles, Vaccination; Vaccination Coverage; Peru (source: MeSH NLM).

\footnotetext{
Universidad Nacional de Cajamarca. Cajamarca, Perú.

Oficina General de Investigación y Transferencia Tecnológica, Instituto Nacional de Salud. Lima, Perú.

Universidad de San Martín de Porres. Lima, Perú.

Licenciada en Enfermería, bachiller en Enfermería; ${ }^{\mathrm{b}}$ licenciada en Enfermería, doctora en Ciencias de Enfermería; ${ }^{\mathrm{c}}$ médico cirujano; ${ }^{\mathrm{d}}$ médico cirujano, maestro en Epidemiología Clínica

El presente estudio forma parte de la tesis: Vásquez-Uriarte K. Vacunación contra el virus del sarampión y predictores de cobertura en menores de cinco años según la encuesta demográfica y de salud familiar, 1996-2017. [tesis para el título de licenciada en Enfermería]. Cajamarca: Facultad de Ciencias de la Salud, Universidad Nacional de Cajamarca; 2019.

Recibido: 11/04/2019 Aprobado: 02/10/2019 En línea: 06/11/2019
}

Citar como: Vásquez-Uriarte K, Ninatanta Ortiz JA, Romani F, Roque-Henriquez JC. Cobertura y factores asociados a la vacunación contra el sarampión en niños de 12 a 59 meses en Perú: estimación basada en la Encuesta Demográfica y de Salud Familiar 2017. Rev Peru Med Exp Salud Publica. 2019;36(4):610-9. doi: 10.17843/ rpmesp.2019.360.4456. 


\section{INTRODUCCIÓN}

La vacuna contra el sarampión es el pilar de la prevención de esta infección viral ${ }^{(1-3)}$. La vacuna administrada a los 12 meses o más genera inmunidad en el $95,0 \%$ de niños, si se agrega una segunda dosis, esta proporción llega al $99,0 \%{ }^{(4,5)}$. Para interrumpir la transmisión endémica y eliminar el sarampión, se necesita un nivel de inmunidad de $93,0 \%$ a $95,0 \%$ en toda la población ${ }^{(5-7)}$. La Organización Mundial de la Salud recomienda que la primera dosis se administre a los 12 meses (nueve meses en países con altas tasas de transmisión del sarampión) y la dosis de refuerzo entre los 15 a 18 meses. En Perú, la primera dosis se aplica a los 12 meses y el refuerzo a los 18 meses ${ }^{(8)}$.

Desde el 2000, Perú no presenta circulación autóctona de sarampión ${ }^{(9)}$, y recién en 2016 las Américas fueron declaradas libres de sarampión. Sin embargo, en 2018 a nivel nacional, fueron notificados 667 casos sospechosos de sarampión, de los cuales 38 fueron confirmados, 16 de ellos provenientes de la región Callao ${ }^{(10)}$. Existirían dos circunstancias que habrían favorecido la reaparición de casos autóctonos de sarampión en Perú: el incremento de la migración internacional, especialmente la proveniente de Venezuela, país que presentó un brote epidémico de sarampión el 2017; y la cobertura de vacunación ${ }^{(9)}$.

Respecto a la cobertura de vacunación contra el sarampión en niños de un año, el Ministerio de Salud del Perú reportó para el 2017 una cobertura de 83\%, con marcadas variaciones entre regiones que van desde $65 \%$ en Arequipa hasta $99 \%$ en Tumbes ${ }^{(9)}$. Esta información proviene de los reportes rutinarios de vacunación de los establecimientos de salud, dicha estimación puede ser susceptible de errores en la compilación de reportes mensuales (especialmente si no se usa registros electrónicos), de retrasos o duplicaciones, además no reporta información del sector privado, y puede sobreestimar la cobertura pues el reporte puede estar vinculada a pagos de incentivos ${ }^{(11)}$. Otro método para estimar la cobertura de vacunación es a través de encuestas poblacionales, como la Encuesta Demográfica y de Salud Familiar (ENDES), que usa muestreos probabilísticos y tiene controles de calidad, por lo que realizaría mejores estimaciones comparada con las coberturas por reportes rutinarios ${ }^{(12)}$.

Por lo descrito, el presente estudio estima en base a una encuesta poblacional, la cobertura de vacunación para la primera dosis y refuerzo contra el sarampión en niños de 12 a 59 meses en Perú. Los resultados del estudio permitirán contar con una estimación confiable de la cobertura de aplicación de esta vacuna, en el contexto de reaparición de casos autóctonos durante el 2018.

\section{MATERIALES Y MÉTODOS}

\section{DISEÑO DE ESTUDIO}

Realizamos un estudio de fuente secundaria basado en la ENDES del 2017, encuesta poblacional de muestreo

\section{MENSAJES CLAVE}

Motivación para realizar el estudio. La vacunación contra el sarampión es fundamental para la prevención de esta infección, por lo cual es importante contar con estimaciones confiables de cobertura.

Principales hallazgos. Según la Encuesta Demográfica y de Salud Familiar 2017, la cobertura a nivel nacional para la primera dosis fue del $70,2 \%$ y para la dosis de refuerzo del $52,0 \%$. Los niños con control de crecimiento y desarrollo en el sector privado tuvieron menores probabilidades de ser vacunados comparado con los del sector público.

Implicancias. La cobertura varió entre regiones; sin embargo, estas fueron menores al 95\%. Lo descrito es un llamado a implementar estrategias para incrementar la vacunación en el sistema de salud.

complejo bietápico, probabilístico de tipo equilibrado, estratificado e independiente para el nivel departamental y por área urbano/rural. La ENDES es realizada por el Instituto Nacional de Estadística e Informática y la base de datos se encuentra disponible de manera libre (http://iinei.inei.gob. pe/microdatos/). El diseño muestral permite inferencias para el nivel nacional y regional, así como para los ámbitos rural y urbano, y para las tres regiones naturales (costa, sierra y selva). La ENDES 2017 fue ejecutada entre marzo y diciembre en una muestra de 35910 viviendas de Perú.

\section{FUENTE DE INFORMACIÓN}

Usamos el cuestionario individual para mujeres de entre 15 a 49 años, dicho cuestionario incluye preguntas sobre características demográficas y sociales, atención prenatal, asistencia del parto, embarazo y lactancia e inmunización de los hijos.

Las unidades de análisis fueron los hijos de las mujeres encuestadas. En caso una mujer haya tenido más de un hijo menor de cinco años, el análisis incluyó a cada uno de ellos. Los criterios de inclusión de las unidades de análisis fueron: niño de 12 a 59 meses de edad (variable Q478 del módulo 70: primera dosis), niño de 18 a 59 meses (variable Q478 del módulo 70: dosis de refuerzo), contar con datos referentes a vacunación contra sarampión (variable H9 o variable S45SP2 del módulo 70).

La ENDES fue la fuente para la información de inmunización contra sarampión; en nuestro análisis, el dato fue considerado válido tuviera o no la fecha de vacunación consignada en la tarjeta de vacunación, esta metodología ha sido previamente usada para evaluar la cobertura de la vacuna de hepatitis $B$ en recién nacidos ${ }^{(13)}$. El uso del dato de vacunación a partir de la tarjeta ha mostrado un mejor rendimiento para estimar cobertura de vacunación (concordancia de $81,0 \%$, sensibilidad $77,0 \%$, especificidad $84,0 \%$ y valor predictivo positivo de $92,0 \%)^{(12)}$. También 
presentamos la estimación de la cobertura según información obtenida de la tarjeta o del reporte materno.

\section{VARIABLES DEL NIÑO}

Las variables dependientes fueron: vacuna de primera dosis contra el sarampión (variable H9) y su refuerzo (variable S45SP), para ambas las categorías de respuesta fueron: vacunado con fecha consignada en la tarjeta, vacunado sin fecha consignada en la tarjeta, no vacunado, vacunado reportado por la madre y no sabe. Cabe precisar que la opción "vacunado reportado por la madre» puede darse en un niño sin tarjeta o con tarjeta de vacunación donde no se consigne la vacuna, incluyendo aquellas recibidas en campañas de vacunación y/o de salud.

Las variables independientes fueron: edad en meses del menor de cinco años (variable Q478), lugar de nacimiento (variable M15 del módulo 69: su domicilio, sector público, sector privado, organismos no gubernamentales, otro), control de crecimiento y desarrollo (variable S466 del módulo 70: no, sí, no sabe), lugar de realización del control de crecimiento y desarrollo (variable S466B del módulo 70: sector público, sector privado, organismos no gubernamentales, otro) y tiene tarjeta de vacunación (variable H1 del módulo 70: sin tarjeta; sí, vista; sí, no vista; ya no tiene tarjeta).

\section{VARIABLES RELACIONADAS A LA MADRE}

Las variables independientes fueron: edad de la madre (variable V102 y V103 del módulo 66), nivel educativo más alto de la madre (variable V106 del módulo 66: no educación, primaria, secundaria, superior), número de controles prenatales (variable M14 del módulo 69), índice de riqueza (variable V190 del módulo 66: más rico, rico, medio, pobre, más pobre), aseguramiento en salud (variables V481, V481D, V481E, V481F, V481G y V481X), región natural (variable SREGION del módulo 66: resto de costa, Lima Metropolitana, sierra, selva), tipo de lugar de residencia (variable V025 del módulo 66: urbano, rural), lugar de residencia de facto (variable V026 del módulo 66: capital, gran ciudad; pequeña ciudad; pueblo; campo) y región política (variable V024 del módulo 66: las 25 regiones del país). Como indicador de migración reciente, se recuperó la variable: años viviendo en el lugar de residencia (variable V104 del módulo 66).

\section{ANÁLISIS ESTADÍSTICO}

Estimamos las frecuencias puntuales de las características de la población de estudio con sus respectivos intervalos de confianza al 95\% (IC95\%). De igual forma estimamos el cumplimiento de la vacunación contra el sarampión (primera dosis y dosis de refuerzo) a nivel nacional, regional y según características sociodemográficas. La comparación de proporciones para muestras independientes se realizó mediante chi cuadrado.
Para el análisis multivariado, fueron consideradas como variables independientes: edad en meses del menor de cinco años, lugar de control de crecimiento y desarrollo, tipo de lugar de residencia, años viviendo en el lugar de residencia y lugar de nacimiento, debido a que en el análisis bivariado presentaron asociación significativa con vacunación contra sarampión (primera dosis o refuerzo). También fueron incluidas en el modelo: nivel educativo más alto de la madre e índice de riqueza debido a su inclusión en modelos multivariados en estudios similares ${ }^{(14,15)}$. Realizamos un análisis de regresión logística considerando como variable dependiente la vacunación de primera dosis contra sarampión y otro modelo para el refuerzo. Calculamos odds ratios (OR) ajustados con IC95\%.

Realizamos el análisis estadístico considerando el muestro complejo de la ENDES. Las variables de ajuste fueron: factor de ponderación (factor mujer V005), número de estrato de la muestra (V022) y número de conglomerado (V001), todos ubicados en el módulo 66. Consideramos como estadísticamente significativo un valor de $\mathrm{p}$ menor de 0,05 . Utilizamos el paquete estadístico Stata versión 15.0 (StataCorp, College Station, Texas, USA). Las bases de datos para el análisis referido a la primera dosis y refuerzo se encuentran en material suplementario.

\section{ASPECTOS ÉTICOS}

Las bases de datos de las ENDES son de acceso público, y respetan la confidencialidad de los participantes. El presente trabajo fue aprobado por la Unidad de Investigación de la Facultad de Ciencias de la Salud de la Universidad Nacional de Cajamarca.

\section{RESULTADOS}

De 21528 niños menores de cinco años, 256 fueron excluidos debido a que no contaban con datos sobre vacunación para la primera dosis y refuerzo, 4003 eran menores de 12 meses de edad y 6233 menores de 18 meses. Finalmente, el análisis para la primera dosis incluyó información de 17269 niños, mientras que la estimación de la cobertura de la dosis de refuerzo se realizó sobre 15039 niños (Figura 1).

Respecto a la portabilidad de la tarjeta de vacunación (sí, vista), esta fue del 77,1\% (IC95\%: 75,8-78,3) para la primera dosis y de 76,1\% (IC95\%: 74,7-77,4) en los niños a quienes le correspondía el refuerzo. La media de edad de las madres fue de 30,5 años (IC95\%: 30,330,8 ), sólo el 36,7\% (IC95\%: 34,9-38,4) de ellas alcanzó el nivel educativo superior y el 88,4\% (IC95\%: 87,4-89,4) refirió haber tenido seis o más controles prenatales. Con relación a los niños, la media de edad fue de 34,9 meses (IC95\%: 34,5-35,2), el 83,0\% (IC95\%: 81,9-84,0) nació en establecimientos públicos de salud. El 66,5\% (IC95\%: $64,9-68,0)$ tuvo control de crecimiento y desarrollo, de 


\section{Primera dosis}

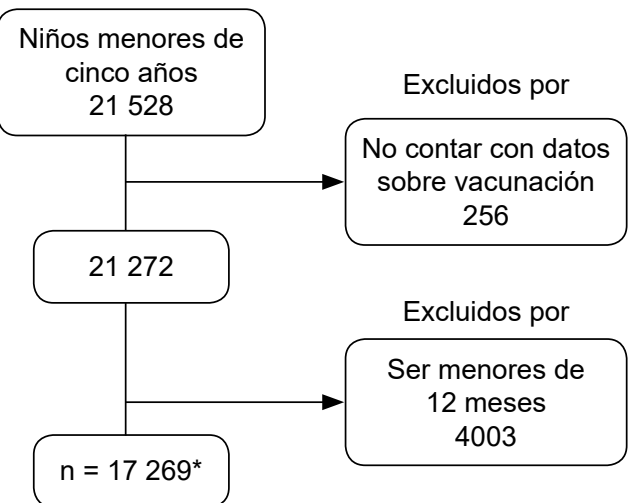

\section{Dosis de refuerzo}

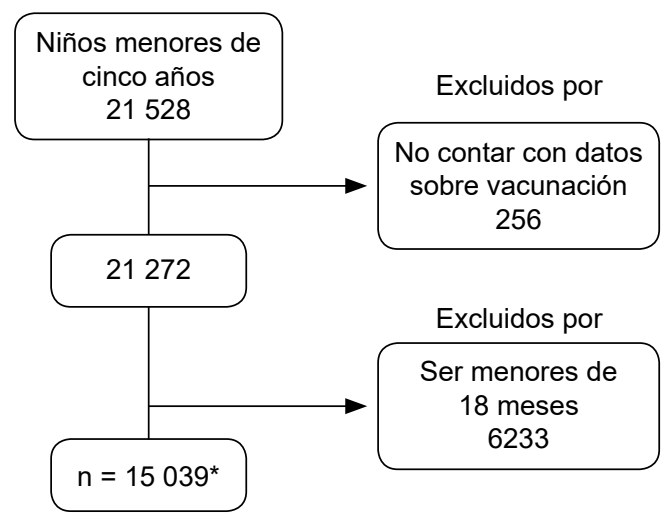

*Constituyen el denominador para el cálculo de la estimación de cobertura de vacunación. La muestra para la dosis de refuerzo se encuentra incluida en la muestra para la primera dosis.

Figura 1. Flujograma de aplicación de los criterios de selección para la conformación de la muestra a partir de la Encuesta Demográfica y de Salud Familiar 2017

los cuales el 96,2\% (IC 95\%: 95,5-96,8) fue realizado en establecimientos públicos de salud. El 79,0\% (IC95\%: 77,6-80,3) contó con seguro de salud (Tabla 1).

\section{ESTIMACIÓN DE COBERTURA A PARTIR DE LA TARJETA DE VACUNACIÓN Y FACTORES ASOCIADOS}

La cobertura nacional para la primera dosis fue de $70,2 \%$ (IC95\%: 68,8-71,6) y para la dosis de refuerzo 52,0\% (IC95\%: $50,5-53,6)$. En el análisis bivariado se encontró asociación estadísticamente significativa para las dos dosis con edad del menor (valor de $p<0,001$ ), contar con control de crecimiento y desarrollo (valor de $p<0,001$ ), lugar de chequeo para control de crecimiento y desarrollo (valor de $p<0,001$ ) y vivir de cinco a más años en el lugar de residencia (valor de $p=0,011$ para primera dosis y valor de $p=0,003$ para refuerzo). Además, para la primera dosis se encontró asociación con tipo de lugar de residencia (valor de $p=0,026$ ) y para la dosis de refuerzo con lugar de nacimiento (valor de $p=0,019)$ (Tabla 1).

Los resultados del análisis multivariado muestran que tras controlar las otras variables predictoras; los niños de 24-35 meses tuvieron más probabilidades de ser vacunados para la primera dosis (OR: 1,59; IC95\%: 1,28-1,97) y dosis de refuerzo (OR: 2,04; IC95\%: 1,62-2,56) comparado con los niños de 12-23 meses y 18-23 meses respectivamente, que los niños con control de crecimiento y desarrollo en el sector privado tuvieron menores probabilidades de ser vacunados para la primera dosis (OR: 0,30; IC95\%: $0,21-0,43$ ) y dosis de refuerzo (OR: 0,26; IC95\%: 0,17$0,40)$ respecto a los controlados en el sector público (Tabla 2).

En el análisis según regiones, para la primera dosis, la estimación puntual más alta la obtuvo Tacna $(78,6 \%)$ y la más baja Junín (55,9\%); para la dosis de refuerzo, la estimación puntual más alta estuvo en Madre de Dios $(67,9 \%)$ y la más baja en Junín $(40,1 \%)$ (Tabla 3$)$.

Tomando en consideración además el reporte de la madre, la cobertura nacional para la primera dosis fue de $87,8 \%$ (IC95\%: 86,9-88,7) y para la dosis de refuerzo 52,2\% (IC95\%: 50,6-53,8). En el análisis según regiones, para la primera dosis y dosis de refuerzo, las estimaciones puntuales más altas las obtuvieron Madre de Dios (93,0\% y 69,0\% respectivamente) y las más bajas Junín (80,8\% y 40,1\% respectivamente) (Tabla 3$)$.

A nivel nacional, para la primera dosis, la diferencia entre la estimación puntual entre la cobertura obtenida a partir de la «tarjeta de vacunación + reporte de la madre» y "tarjeta de vacunación» fue de 17,6 puntos porcentuales ( $p p)$, dicha diferencia para la dosis de refuerzo fue de 0,2 pp. A nivel de regiones el rango de tales diferencias para la primera dosis fue de 11,3 a 24,9 pp y para la dosis de refuerzo de 0,0 a 0,6 pp (Tabla 3).

\section{DISCUSIÓN}

En nuestro estudio, el 70,2\% de niños peruanos de 12 a 59 meses de edad que participaron en la ENDES 2017 recibieron la primera dosis de la vacuna contra el sarampión, mientras que sólo la mitad de niños habría recibido la dosis de refuerzo a partir de los 18 meses de edad. Estas estimaciones de cobertura incluyen información de vacunación del niño menor de cinco años, y es una forma de estimación usada en otros estudios basados en encuestas poblacionales ${ }^{(14-18)}$. Las coberturas estimadas estarían por debajo del 95,0\% establecidos para la primera dosis y refuerzo a nivel nacional, lo cual es parte del objetivo a ser alcanzado al $2020{ }^{(3)}$. 
Tabla 1. Características sociodemográficas y proporción de vacunación contra el sarampión en hijos menores de cinco años de mujeres enroladas en la Encuesta Demográfica y de Salud Familiar 2017

\begin{tabular}{|c|c|c|c|c|c|c|}
\hline \multirow{3}{*}{ Característica } & \multicolumn{3}{|c|}{$\begin{array}{c}\text { Primera dosis } \\
(n=17269)\end{array}$} & \multicolumn{3}{|c|}{$\begin{array}{c}\text { Dosis de refuerzo } \\
(n=15039)\end{array}$} \\
\hline & $\begin{array}{l}\text { Proporción } \\
\text { según } \\
\text { característica }\end{array}$ & $\begin{array}{l}\text { Cobertura de } \\
\text { primera dosis * } \\
\text { (12 a } 59 \text { meses) }\end{array}$ & $\begin{array}{l}\text { Valor } \\
\text { de } p^{\dagger}\end{array}$ & $\begin{array}{l}\text { Proporción } \\
\text { según } \\
\text { característica }\end{array}$ & $\begin{array}{l}\text { Cobertura de } \\
\text { refuerzo * } \\
\text { (18 a } 59 \text { meses) }\end{array}$ & $\begin{array}{l}\text { Valor } \\
\text { de } p^{\dagger}\end{array}$ \\
\hline & $\%(I C 95 \%)$ & $\%($ IC95\%) & & $\%$ (IC95\%) & $\%$ (IC95\%) & \\
\hline Edad de la madre (años) & & & 0,461 & & & 0,701 \\
\hline $15-19$ & $14,8(13,9-15,8)$ & $70,7(67,4-73,8)$ & & $14,7(13,7-15,8)$ & $52,1(48,4-55,8)$ & \\
\hline $20-24$ & $15,1(14,1-16,1)$ & $68,5(65,6-71,3)$ & & $15,2(14,2-16,3)$ & $51,0(47,5-54,4)$ & \\
\hline $25-29$ & $14,9(14,0-15,9)$ & $71,8(69,0-74,4)$ & & $15,2(14,2-16,2)$ & $54,6(51,2-57,9)$ & \\
\hline $30-34$ & $15,2(14,2-16,3)$ & $68,8(65,0-72,2)$ & & $15,1(14,1-16,2)$ & $52,4(48,5-56,3)$ & \\
\hline $35-39$ & $15,2(14,2-16,3)$ & $72,4(68,8-75,8)$ & & $15,2(14,1-16,4)$ & $52,1(48,2-56,0)$ & \\
\hline $40-44$ & $12,6(11,6-13,7)$ & $68,4(64,2-72,4)$ & & $12,4(11,3-13,5)$ & $52,2(47,9-56,5)$ & \\
\hline $45-49$ & $12,1(11,2-13,0)$ & $71,0(66,8-74,8)$ & & $12,2(11,3-13,2)$ & $49,4(44,8-53,9)$ & \\
\hline $\begin{array}{l}\text { Nivel educativo más alto } \\
\text { de la madre }\end{array}$ & & & 0,941 & & & 0,390 \\
\hline Superior & $36,7(34,9-38,4)$ & $70,4(68,0-72,7)$ & & $36,5(34,6-38,3)$ & $51,2(48,5-54,0)$ & \\
\hline Secundaria & $44,3(42,7-45,9)$ & $69,9(67,8-71,9)$ & & $44,3(42,7-46,0)$ & $52,9(50,7-55,2)$ & \\
\hline Primaria & $17,3(16,1-18,6)$ & $70,8(68,1-73,3)$ & & $17,5(16,3-18,9)$ & $52,0(48,8-55,2)$ & \\
\hline No educación & $1,7(1,4-2,0)$ & $70,0(63,1-76,2)$ & & $1,7(1,4-2,0))$ & $45,4(37,7-53,3)$ & \\
\hline $\begin{array}{l}\text { Controles prenatales } \\
\text { ( } \geq 6 \text { controles) }\end{array}$ & & & 0,397 & & & 0,291 \\
\hline Sí & $88,4(87,4-89.4)$ & $70,8(69,3-72,2)$ & & $88,5(87,4-89,6)$ & $52,7(51,0-54,4)$ & \\
\hline No & $11,2(10,2-12,2)$ & $68,6(64,3-72,6)$ & & $11,1(10,1-12,3)$ & $50,1(45,0-55,1)$ & \\
\hline No sabe & $0,4(0,3-0,6)$ & $77,7(60,0-89,0)$ & & $0,4(0,2-0,6)$ & $67,4(44,1-84,4)$ & \\
\hline $\begin{array}{l}\text { Edad del menor de cinco } \\
\text { años (meses) }\end{array}$ & & & $<0,001$ & & & $<0,001$ \\
\hline $12-23(18-23)$ & $26,7(25,6-27,9)$ & $68,6(66,1-71,1)$ & & $16,1(15,1-17,2)$ & $41,3(37,8-44,9)$ & \\
\hline $24-35$ & $24,6(23,5-25,7)$ & $75,1(72,5-77,5)$ & & $28,2(26,9-29,4)$ & $54,9(51,8-58,0)$ & \\
\hline $36-47$ & $25,5(24,3-26,7)$ & $70,3(67,6-72,8)$ & & $29,2(27,9-30,6)$ & $52,8(50,0-55,5)$ & \\
\hline $48-59$ & $23,1(22,1-24,2)$ & $66,9(64,2-69,5)$ & & $26,5(25,3-27,7)$ & $54,7(51,9-57,5)$ & \\
\hline Lugar de nacimiento & & & 0,178 & & & 0,019 \\
\hline Su domicilio & $6,3(5,7-7,0)$ & $69,7(64,8-74,3)$ & & $6,1(5,5-6,8)$ & $52,6(46,5-58,7)$ & \\
\hline Sector público & $83,0(81,9-84,0)$ & $70,8(69,0-72,3)$ & & $82,6(81,4-83,6)$ & $52,7(51,0-54,3)$ & \\
\hline Sector privado & $9,5(8,7-10,4)$ & $65,7(61,0-70,1)$ & & $10,1(9,2-11,0)$ & $45,5(41,1-50,0)$ & \\
\hline $\begin{array}{l}\text { Organismos no } \\
\text { gubernamentales }\end{array}$ & $0,2(0,1-0,4)$ & $76,0(53,1-89,9)$ & & $0,2(0,1-0,4)$ & $41,2(17,6-69,6)$ & \\
\hline Otro & $1,0(0,7-1,3)$ & $71,6(58,4-81,9)$ & & $1,0(0,7-1,4)$ & $62,6(48,6-74,8)$ & \\
\hline $\begin{array}{l}\text { Control de crecimiento y } \\
\text { desarrollo }\end{array}$ & & & $<0,001$ & & & $<0,001$ \\
\hline Sí & $66,5(64,9-68,0)$ & $79,2(77,6-80,6)$ & & $63,2(61,5-64,8)$ & $61,9(60,0-63,8)$ & \\
\hline No & $33,3(31,8-34,8)$ & $52,9(50,4-55,5)$ & & $36,6(34,9-38,2)$ & $35,4(33,0-37,9)$ & \\
\hline No sabe & $0,2(0,2-0,4)$ & $3,1(0,7-13,3)$ & & $0,3(0,2-0,4)$ & $0,0(0,0)$ & \\
\hline $\begin{array}{l}\text { Lugar de control de } \\
\text { crecimiento y desarrollo }\end{array}$ & & & $<0,001$ & & & $<0,001$ \\
\hline Sector público & $96,2(95,5-96,8)$ & $80,0(78,4-81,4)$ & & $95,9(95,1-96,6)$ & $63,0(61,1-64,9)$ & \\
\hline Sector privado & $3,1(2,5-3,7)$ & $55,6(47,2-63,6)$ & & $3,3(2,7-4,0)$ & $32,4(24,0-42,1)$ & \\
\hline $\begin{array}{l}\text { Organismos no } \\
\text { gubernamentales }\end{array}$ & $0,1(0,1-0,3)$ & $59,7(26,2-86,1)$ & & $0,2(0,1-0,3)$ & $51,4(19,5-82,2)$ & \\
\hline Otro & $0,6(0,4-1,0)$ & $75,9(55,3-88,9)$ & & $0,6(0,4-1,1)$ & $46,2(23,0-71,2)$ & \\
\hline
\end{tabular}

* Sí: vacunado (con fecha) + vacunado (sin fecha), consignadas en la tarjeta para la primera dosis o refuerzo; No: no vacunado + vacunado reportado por la madre + no sabe

+ Prueba de chi cuadrado

IC95\%: intervalo de confianza al 95\%

(Continuá en la página 615) 
Tabla 1. Características sociodemográficas y proporción de vacunación contra el sarampión en hijos menores de cinco años de mujeres enroladas en la Encuesta Demográfica y de Salud Familiar 2017 (Viene de la página 614)

\begin{tabular}{|c|c|c|c|c|c|c|}
\hline \multirow{3}{*}{ Característica } & \multicolumn{3}{|c|}{$\begin{array}{l}\text { Primera dosis } \\
(n=17269)\end{array}$} & \multicolumn{3}{|c|}{$\begin{array}{l}\text { Dosis de refuerzo } \\
(n=15039)\end{array}$} \\
\hline & $\begin{array}{c}\text { Proporción } \\
\text { según } \\
\text { característica }\end{array}$ & $\begin{array}{c}\text { Cobertura de } \\
\text { primera dosis * } \\
\text { (12 a } 59 \text { meses) }\end{array}$ & $\begin{array}{l}\text { Valor } \\
\text { de } p^{\dagger}\end{array}$ & $\begin{array}{l}\text { Proporción } \\
\text { según } \\
\text { característica }\end{array}$ & $\begin{array}{c}\text { Cobertura de } \\
\text { refuerzo * } \\
\text { (18 a } 59 \text { meses) }\end{array}$ & $\begin{array}{l}\text { Valor } \\
\text { de } p^{\dagger}\end{array}$ \\
\hline & $\%$ (IC95\%) & $\%$ (IC95\%) & & $\%$ (IC95\%) & $\%$ (IC95\%) & \\
\hline Índice de riqueza & & & 0,394 & & & 0,474 \\
\hline Más rico & $21,0(18,8-23,4)$ & $70,7(66,6-74,4)$ & & $20,9(18,7-23,3)$ & $50,5(46,3-54,6)$ & \\
\hline Rico & $21,0(19,3-22,7)$ & $70,5(67,5-73,3)$ & & $21,3(19,5-23,1)$ & $51,2(47,7-54,8)$ & \\
\hline Medio & $19,9(18,5-21,4)$ & $68,2(65,4-70,8)$ & & $19,9(18,4-21,5)$ & $51,2(48,0-54,3)$ & \\
\hline Pobre & $19,6(18,1-21,2)$ & $69,8(67,3-72,2)$ & & $19,4(17,9-21,0)$ & $53,7(50,6-56,8)$ & \\
\hline Más pobre & $18,6(17,2-20,0)$ & $72,2(70,1-74,2)$ & & $18,5(17,1-20,0)$ & $53,9(51,5-56,3)$ & \\
\hline \multicolumn{7}{|l|}{ Cuenta con seguro de salud } \\
\hline Seguro privado & & & 0,414 & & & 0,352 \\
\hline Sí & $1,4(1,0-2,0)$ & $75,8(61,2-86,1)$ & & $1,4(1,0-2,0)$ & $59,8(43,1-74,4)$ & \\
\hline No & $98,6(98,0-99,0)$ & $70,2(68,7-71,6)$ & & $98,6(98,0-99,0)$ & $51,9(50,4-53,5)$ & \\
\hline EsSalud/IPSS & & & 0,457 & & & 0,269 \\
\hline Sí & $24,1(22,6-25,6)$ & $71,1(68,2-73,9)$ & & $24,1(22,5-25,7)$ & $50,5(47,2-53,8)$ & \\
\hline No & $75,9(74,4-77,4)$ & $70,0(68,4-71,4)$ & & $75,9(74,3-77,5)$ & $52,5(50,8-54,2)$ & \\
\hline Fuerzas armadas o policiales & & & 0,665 & & & 0,177 \\
\hline Sí & $1,1(0,8-1,6)$ & $66,5(47,3-81,5)$ & & $1,0(0,7-1,5)$ & $40,7(25,9-57,4)$ & \\
\hline No & $98,9(98,4-99,2)$ & $70,3(68,8-71,7)$ & & $99,0(98,5-99,3)$ & $52,2(50,6-53,7)$ & \\
\hline Seguro Integral de Salud (SIS) & & & 0,344 & & & 0,283 \\
\hline Sí & $52,7(50,6-54,7)$ & $69,6(67,8-71,4)$ & & $52,7(50,7-54,8)$ & $52,8(50,8-54,8)$ & \\
\hline No & $47,3(45,3-49,4)$ & $70,9(68,8-73,0)$ & & $47,3(45,2-49,3)$ & $51,2(48,8-53,5)$ & \\
\hline Entidad prestadora de salud & & & 0,616 & & & 0,834 \\
\hline Sí & $2,1(1,7-2,8)$ & $73,1(60,8-82,6)$ & & $2,2(1,7-2,9)$ & $50,6(37,2-63,9)$ & \\
\hline No & $97,9(97,2-98,3)$ & $70,2(68,7-71,6)$ & & $97,8(97,1-98,3)$ & $52,1(50,5-53,6)$ & \\
\hline Región natural & & & 0,723 & & & 0,501 \\
\hline Lima Metropolitana & $33,8(30,3-37,6)$ & $70,0(66,5-73,3)$ & & $34,1(30,6-37,9)$ & $50,8(47,1-54,4)$ & \\
\hline Resto de costa & $23,3(21,0-25,8)$ & $69,8(67,5-72,0)$ & & $23,1(20,8-25,6)$ & $52,2(49,7-54,7)$ & \\
\hline Selva & $12,4(11,0-14,0)$ & $69,3(66,8-71,8)$ & & $12,4(10,9-14,0)$ & $54,2(51,1-57,3)$ & \\
\hline Sierra & $30,4(27,9-33,1)$ & $71,2(69,3-73,1)$ & & $30,4(27,8-33,0)$ & $52,4(50,3-54,5)$ & \\
\hline Tipo de lugar de residencia & & & 0,026 & & & 0,126 \\
\hline Urbano & $79,3(77,8-80.8)$ & $69,6(67,9-71,3)$ & & $79,3(77,8-80,8)$ & $51,5(49,7-53,4)$ & \\
\hline Rural & $20,7(19,2-22,2)$ & $72,6(70,6-74,5)$ & & $20,7(19,2-22,2)$ & $53,9(51,5-56,3)$ & \\
\hline Lugar de residencia de facto & & & 0,219 & & & 0,430 \\
\hline Capital, gran ciudad & $33,8(30,3-37,6)$ & $70,0(66,5-73,3)$ & & $34,1(30,6-37,9)$ & $50,8(47,1-54,4)$ & \\
\hline Pequeña ciudad & $24,5(22,3-26,8)$ & $70,3(68,1-72,4)$ & & $24,1(21,9-26,4)$ & $51,6(49,2-53,9)$ & \\
\hline Pueblo & $21,0(19,5-22,6)$ & $68,3(65,9-70,6)$ & & $21,1(19,6-22,7)$ & $52,7(50,1-55,4)$ & \\
\hline Campo & $20,7(19,2-22,2)$ & $72,6(70,6-74,5)$ & & $20,7(19,2-22,2)$ & $53,9(51,5-56,3)$ & \\
\hline $\begin{array}{l}\text { Años viviendo en el lugar de } \\
\text { residencia }\end{array}$ & & & 0,011 & & & 0,003 \\
\hline$<5$ & $16,5(15,5-17,6)$ & $66,4(63,1-69,5)$ & & $18,3(17,2-19,4)$ & $47,0(43,5-50,6)$ & \\
\hline$\geq 5$ & $83,5(82,4-84,5)$ & $70,7(69,1-72,3)$ & & $81,7(80,6-82,8)$ & $53,0(51,2-54,7)$ & \\
\hline Tiene tarjeta de vacunación & & & $<0,001$ & & & $<0,001$ \\
\hline Sin tarjeta & $0,7(0,5-0,9)$ & $9,5(4,9-17,6)$ & & $0,6(0,4-0,9)$ & $2,6(1,1-6,5)$ & \\
\hline Sí, vista & $77,1(75,8-78,3)$ & $90,5(89,5-91,3)$ & & $76,1(74,7-77,4)$ & $68,0(66,3-69,7)$ & \\
\hline Sí, no vista & $18,2(17,1-19,5)$ & $2,0(1,4-2,8)$ & & $18,9(17,7-20,2)$ & $1,1(0,7-1,8)$ & \\
\hline Ya no tiene tarjeta & $4,0(3,5-4,6)$ & $2,5(1,1-5,5)$ & & $4,4(3,8-5,1)$ & $1,8(0,6-5,4)$ & \\
\hline
\end{tabular}


Tabla 2. Características asociadas a la vacunación contra el sarampión en hijos menores de cinco años de mujeres enroladas en la Encuesta Demográfica y de Salud Familiar 2017

\begin{tabular}{|c|c|c|c|c|c|c|}
\hline \multirow[t]{2}{*}{ Característica } & \multicolumn{3}{|c|}{$\begin{array}{c}\text { Primera dosis * }(n=17269) \\
(12 \text { a } 59 \text { meses })\end{array}$} & \multicolumn{3}{|c|}{$\begin{array}{l}\text { Refuerzo * }(n=15039) \\
(18 \text { a } 59 \text { meses })\end{array}$} \\
\hline & ORa & IC95\% & Valor de $p$ & ORa & IC95\% & Valor de $\mathrm{p}$ \\
\hline \multicolumn{7}{|c|}{ Nivel educativo más alto de la madre } \\
\hline Superior & Ref & & & Ref & & \\
\hline Secundaria & 1,01 & $0,83-1,23$ & 0,906 & 1,14 & $0,93-1,40$ & 0,200 \\
\hline Primaria & 0,90 & $0,68-1,20$ & 0,465 & 0,95 & $0,72-1,25$ & 0,708 \\
\hline No educación & 0,73 & $0,44-1,21$ & 0,222 & 0,63 & $0,39-1,03$ & 0,068 \\
\hline \multicolumn{7}{|c|}{ Edad del menor de cinco años (meses) } \\
\hline $12-23(18-23)$ & Ref & & & Ref & & \\
\hline $24-35$ & 1,59 & $1,28-1,97$ & $<0,001$ & 2,04 & $1,62-2,56$ & $<0,001$ \\
\hline $36-47$ & 1,21 & $0,95-1,54$ & 0,129 & 1,75 & $1,40-2,20$ & $<0,001$ \\
\hline $48-59$ & 1,20 & $0,96-1,51$ & 0,116 & 2,32 & $1,84-2,94$ & $<0,001$ \\
\hline \multicolumn{7}{|c|}{ Lugar de nacimiento } \\
\hline Sector público & Ref & & & Ref & & \\
\hline Su domicilio & 0,94 & $0,68-1,31$ & 0,718 & 0,88 & $0,63-1,24$ & 0,472 \\
\hline Sector privado & 0,80 & $0,58-1,09$ & 0,161 & 0,72 & $0,57-0,92$ & 0,009 \\
\hline ONG & 0,63 & $0,23-1,71$ & 0,360 & 1,16 & $0,29-4,62$ & 0,832 \\
\hline \multicolumn{7}{|c|}{ Lugar de control de crecimiento y desarrollo } \\
\hline Sector público & Ref & & & Ref & & \\
\hline Sector privado & 0,30 & $0,21-0,43$ & $<0,001$ & 0,26 & $0,17-0,40$ & $<0,001$ \\
\hline ONG & 0,38 & $0,09-1,62$ & 0,190 & 0,57 & $0,13-2,49$ & 0,453 \\
\hline \multicolumn{7}{|l|}{ Índice de riqueza } \\
\hline Más rico & Ref & & & Ref & & \\
\hline Rico & 0,99 & $0,73-1,36$ & 0,971 & 1,10 & $0,83-1,45$ & 0,524 \\
\hline Medio & 0,77 & $0,55-1,07$ & 0,122 & 1,00 & $0,77-1,30$ & 0,988 \\
\hline Pobre & 0,90 & $0,64-1,25$ & 0,515 & 1,15 & $0,86-1,53$ & 0,350 \\
\hline Más pobre & 0,77 & $0,51-1,17$ & 0,219 & 1,13 & $0,77-1,64$ & 0,538 \\
\hline \multicolumn{7}{|c|}{ Tipo de lugar de residencia } \\
\hline Urbano & Ref & & & Ref & & \\
\hline Rural & 1,41 & $1,08-1,83$ & 0,011 & 1,04 & $0,79-1,36$ & 0,777 \\
\hline \multicolumn{7}{|c|}{ Años viviendo en el lugar de residencia } \\
\hline$<5$ & Ref & & & Ref & & \\
\hline$\geq 5$ & 1,23 & $0,99-1,53$ & 0,064 & 1,37 & $1,12-1,69$ & 0,003 \\
\hline
\end{tabular}

* Sí: vacunado (con fecha) + vacunado (sin fecha), consignadas en la tarjeta para la primera dosis o refuerzo; No: no vacunado + vacunado reportado por la madre + no sabe

ONG: Organismos no gubernamentales, ORa: Odds ratio ajustado, IC95\%: intervalo de confianza al $95 \%$

Ref: categoría de referencia

Nuestras estimaciones difieren de lo generado por reportes rutinarios del 2017 de los establecimientos de salud del Perú, según estos, el $83,0 \%$ de los niños de un año había recibido la primera dosis, y el 66,0\% de los niños de 18 meses había recibido el refuerzo ${ }^{(19)}$. Las diferencias se explican por los métodos de estimación empleados; pues como estudios previos lo señalan, la estimación a partir de reportes rutinarios pueden sobreestimar la cobertura de vacunación ${ }^{(12)}$.

La mayoría de los estudios que han estimado coberturas de vacunación contra sarampión usando las encuestas demográficas y de salud (DHS) fueron realizados en países africanos. En Senegal fueron realizados dos estudios, los cuales reportan coberturas de $82,1 \%$ en 2199 niños de 12 a 23 meses (DHS 2010-2011) ${ }^{(15)}$ y 79,2\% en 2444 niños de 12 a 23 meses (DHS 2013-2014) (20); en el Congo se reportó una cobertura de $70,0 \%$ en 6947 niños de 6 a 59 meses (DHS 2013-2014) (16); en Etiopía la cobertura fue de 55,7\% en 1927 niños de 12 a 23 meses (DHS 2011) ${ }^{(17) ; ~ p o r ~ u ́ l t i m o, ~ e n ~}$ Pakistán se reportó $62,5 \%$ de cobertura en 9177 niños de 0 a 60 meses (DHS 2006-2007) ${ }^{(14)}$. Todas las estimaciones presentadas consideraron tanto el recuerdo de la madre, como el obtenido de las tarjetas de vacunación, en nuestro estudio considerando dicha forma de estimación la cobertura nacional tuvo 17,6 puntos porcentuales más para la primera dosis, mientras que para el refuerzo la variación fue mínima, con menos de un punto porcentual de diferencia.

La meta en los planes internacionales establece que al menos el $80,0 \%$ de los municipios (o su equivalente) de un 
Tabla 3. Cumplimiento de la vacunación contra el sarampión en menores de cinco años de madres enroladas en la Encuesta Demográfica y de Salud Familiar 2017, según región política

\begin{tabular}{|c|c|c|c|c|c|c|}
\hline \multirow{3}{*}{ Región Política } & \multicolumn{2}{|c|}{$\begin{array}{l}\text { Registro a partir de tarjeta de } \\
\text { vacunación * }\end{array}$} & \multicolumn{2}{|c|}{$\begin{array}{l}\text { Registro a partir de tarjeta de } \\
\text { vacunación + reporte de madre }\end{array}$} & \multicolumn{2}{|c|}{$\begin{array}{c}\text { Diferencias entre } \\
\text { estimaciones } \\
\text { puntuales }\end{array}$} \\
\hline & $\begin{array}{c}\text { Primera dosis } \\
\text { ( } n=17269) \\
(12 \text { a } 59 \text { meses) } \\
\text { (a) }\end{array}$ & $\begin{array}{c}\text { Refuerzo } \\
(n=15039) \\
(18 \text { a } 59 \text { meses) } \\
\text { (c) }\end{array}$ & $\begin{array}{c}\text { Primera dosis } \\
\text { ( } n=17 \text { 269) } \\
(12 \text { a } 59 \text { meses) } \\
\text { (b) }\end{array}$ & $\begin{array}{c}\text { Refuerzo } \\
(\mathrm{n}=15 \text { 039) } \\
(18 \text { a } 59 \text { meses) } \\
\text { (d) }\end{array}$ & $(b-a)$ & $(d-c)$ \\
\hline & $\%(I C 95 \%)$ & $\%(I C 95 \%)$ & $\%(I C 95 \%)$ & $\%(I C 95 \%)$ & $\%$ & $\%$ \\
\hline Amazonas & $73,8(69,8-77,5)$ & $59,5(54,6-64,2)$ & $89,7(86,7-92,1)$ & $59,6(54,7-64,3)$ & 15,9 & 0,1 \\
\hline Ancash & $66,9(62,7-70,9)$ & $49,9(45,1-54,7)$ & $86,3(82,9-89,1)$ & $49,9(45,1-54,7)$ & 19,4 & 0,0 \\
\hline Apurímac & $69,0(64,7-73,0)$ & $49,1(44,8-53,5)$ & $87,8(84,6-90,3)$ & $49,4(45,1-53,8)$ & 18,8 & 0,3 \\
\hline Arequipa & $73,4(69,1-77,3)$ & $53,1(48,6-57,6)$ & $90,7(87,6-93,0)$ & $53,2(48,6-57,6)$ & 17,3 & 0,1 \\
\hline Ayacucho & $69,3(64,1-74,0)$ & $46,3(41,7-51,1)$ & $85,0(81,4-88,0)$ & $46,4(41,8-51,2)$ & 15,7 & 0,1 \\
\hline Cajamarca & $73,5(69,1-77,5)$ & $57,2(52,3-62,0)$ & $89,9(87,3-91,9)$ & $57,6(52,7-62,4)$ & 16,4 & 0,4 \\
\hline Callao & $63,0(58,8-67,0)$ & $48,1(43,5-52,7)$ & $86,0(82,2-89,1)$ & $48,1(43,5-52,7)$ & 23,0 & 0,0 \\
\hline Cusco & $68,6(61,5-74,9)$ & $51,3(43,4-59,1)$ & $81,7(75,9-86,3)$ & $51,3(43,4-59,1)$ & 13,1 & 0,0 \\
\hline Huancavelica & $75,0(69,3-80,0)$ & $52,1(46,1-58,0)$ & $86,3(81,6-89,9)$ & $52,4(46,3-58,4)$ & 11,3 & 0,3 \\
\hline Huánuco & $68,6(63,3-73,4)$ & $48,0(41,0-55,0)$ & $90,4(87,4-92,8)$ & $48,0(41,0-55,0)$ & 21,8 & 0,0 \\
\hline Ica & $73,9(68,1-79,0)$ & $59,7(52,5-66,5)$ & $92,6(89,3-94,9)$ & $59,8(52,6-66,7)$ & 18,7 & 0,1 \\
\hline Junín & $55,9(49,4-62,3)$ & $40,1(32,7-47,9)$ & $80,8(74,4-86,0)$ & $40,1(32,7-47,9)$ & 24,9 & 0,0 \\
\hline La Libertad & $65,1(59,8-70,0)$ & $47,1(41,8-52,4)$ & $88,6(85,2-91,4)$ & $47,1(41,8-52,4)$ & 23,5 & 0,0 \\
\hline Lambayeque & $75,8(70,4-80,4)$ & $60,0(53,0-66,5)$ & $90,9(87,0-93,7)$ & $61,4(54,4-68,0)$ & 15,1 & 0,4 \\
\hline Lima & $70,7(66,8-74,3)$ & $50,9(46,9-54,9)$ & $87,5(84,9-89,6)$ & $50,9(46,9-54,9)$ & 16,8 & 0,0 \\
\hline Loreto & $76,7(71,9-80,8)$ & $62,2(55,9-68,0)$ & $90,9(87,4-93,5)$ & $62,2(55,9-68,0)$ & 14,2 & 0,0 \\
\hline Madre de Dios & $78,2(72,6-82,9)$ & $67,9(62,0-73,3)$ & $93,0(88,9-95,7)$ & $69,0(63,4-74,1)$ & 14,8 & 1,1 \\
\hline Moquegua & $72,5(65,9-78,3)$ & $51,3(44,5-58,1)$ & $89,8(85,4-93,0)$ & $51,3(44,5-58,1)$ & 17,3 & 0,0 \\
\hline Pasco & $73,8(68,1-78,8)$ & $60,1(53,5-66,4)$ & $86,6(81,8-90,3)$ & $60,1(53,5-66,4)$ & 12,8 & 0,0 \\
\hline Piura & $71,6(67,2-75,7)$ & $49,7(45,1-54,4)$ & $87,4(84,2-90,1)$ & $50,2(45,6-54,8)$ & 15,8 & 0,5 \\
\hline Puno & $77,9(70,0-84,3)$ & $65,0(56,6-72,6)$ & $89,6(84,2-93,3)$ & $65,0(56,6-72,6)$ & 11,7 & 0,0 \\
\hline San Martín & $67,5(61,7-73,0)$ & $50,2(43,7-56,8)$ & $87,8(83,8-91,0)$ & $50,8(44,5-57,2)$ & 20,3 & 0,6 \\
\hline Tacna & $78,6(73,5-83,0)$ & $64,8(58,6-70,6)$ & $90,7(87,3-93,2)$ & $64,8(58,6-70,6)$ & 12,1 & 0,0 \\
\hline Tumbes & $65,6(59,9-70,8)$ & $45,6(39,7-51,7)$ & $81,4(76,2-85,6)$ & $45,6(39,7-51,7)$ & 15,8 & 0,0 \\
\hline Ucayali & $67,2(62,4-71,6)$ & $51,7(46,4-57,1)$ & $89,3(86,2-91,8)$ & $51,8(46,4-57,2)$ & 22,1 & 0,1 \\
\hline Total & $70,2(68,8-71,6)$ & $52,0(50,5-53,6)$ & $87,8(86,9-88,7)$ & $52,2(50,6-53,8)$ & 17,6 & 0,2 \\
\hline
\end{tabular}

*Sí: vacunado (con fecha) + vacunado (sin fecha), consignadas en la tarjeta para la primera dosis o refuerzo; No: no vacunado + vacunado reportado por la madre + no sabe

† Sí: vacunado (con fecha) + vacunado (sin fecha) + reportado por la madre, para la primera dosis o refuerzo; No: no vacunado + no sabe IC95\%: intervalo de confianza al $95 \%$

país alcancen una cobertura de $95,0 \%$ o más para cada una de las dosis ${ }^{(3,19)}$. Si bien, no mostramos coberturas a nivel de distrito, sino a nivel regional, el estudio encontró que ninguna región peruana alcanzó para la primera dosis y dosis de refuerzo una cobertura de $95,0 \%$. Encontramos coberturas variables entre regiones, y sólo las regiones de Arequipa, Huánuco, Ica, Lambayeque, Loreto, Madre de Dios y Tacna superan el $90,0 \%$ si consideramos información obtenida tanto de la tarjeta de vacunación como por reporte de la madre.

En nuestro estudio usamos como numerador a los niños de 12 a 59 meses y de 18 a 59 meses de edad en quienes se pudo constatar la primera dosis y dosis de refuerzo contra el sarampión a partir de la tarjeta de vacunación (con o sin fecha consignada). Por otro lado, el denominador incluyó para la primera dosis y refuerzo a todos los niños de los grupos etarios indicados previamente. Esta definición permite reducir la posibilidad de sesgos de mala clasificación. No pudimos evaluar la oportunidad de la vacunación (edad exacta de aplicación), es decir, si dichas vacunaciones fueron realizadas, según norma técnica nacional, a los 12 y 18 meses. Con esta consideración, los estimados de cobertura brindados no consideran el componente de oportunidad, lo cual ha sido abordado en otros estudios ${ }^{(14,18)}$.

Encontramos que, entre aquellos niños con controles de crecimiento y desarrollo en el sector público, y que residen en áreas rurales tienen mayor probabilidad de contar con 
vacunación de sarampión en su primera dosis. Los factores independientemente asociados con tener dosis de refuerzo fueron el tener controles de crecimiento y desarrollo en sector público, y el residir de cinco a más años en su domicilio actual. También encontramos que el nacer en establecimientos de salud privados se encuentra asociado al no uso de la dosis de refuerzo. Es decir que en aquellos niños que acuden a sus controles de crecimiento y desarrollo en establecimientos públicos, la probabilidad de recibir las dos dosis de vacuna contra sarampión es mayor, este factor podría estar asociado, a su vez, con residir cinco o más años en su domicilio actual, lo cual permitiría un acceso sostenido a los establecimientos de salud, especialmente los públicos, así como una mayor fidelidad a sus servicios, incluyendo los controles de crecimiento y desarrollo, y la vacunación.

Un estudio con metodología similar aplicado a la vacunación contra hepatitis $B$ en recién nacidos encontró que los factores vinculados a la vacunación fueron el acceso al parto institucional, y los asociados a este último como el aseguramiento ${ }^{(13)}$. La vacuna del recién nacido contra hepatitis $B$ debe ser colocada en las primeras 24 horas de vida, lo cual, es propiciado cuando el parto se da en el establecimiento de salud. Por el contrario, la vacuna contra sarampión en su primera dosis debe ser aplicada recién al año, por lo que para hacer efectiva la vacunación, el niño debe acudir al establecimiento de salud en dichos meses de vida, lo cual puede asociarse con el contar con controles de crecimiento y desarrollo, y con tener un lugar de residencia permanente.

Estudios realizados en encuestas poblacionales han encontrado que tanto la vacunación contra sarampión ${ }^{(16)}$, así como la vacunación completa ${ }^{(14,15,17)}$ estuvieron asociadas a un mejor quintil de riqueza. En nuestro estudio no se encontró dicha asociación estadística, por el contrario, un antecedente que evaluó la cobertura de vacunación con hepatitis $B$ al nacimiento en el Perú, reportó que entre aquellos con menor índice de riqueza las coberturas de vacunación fueron mejores ${ }^{(13)}$. Otro factor que ha sido asociado a vacunación completa es un mejor nivel educativo de la madre ${ }^{(14,15,17)}$, sin embargo, tampoco encontramos asociación entre nivel educativo y cobertura de vacunación en la primera dosis y refuerzo. Por último, la realización de controles prenatales también ha sido asociada con el cumplimiento del esquema de vacunación ${ }^{(15)}$, lo cual tampoco ha sido encontrado en el presente análisis.

El estudio presentó las siguientes limitaciones: no brinda estimaciones de coberturas anuales que nos permitan comparar con los resultados de otros estudios o reportes que si lo hacen; al ser un estudio de fuente secundaria sólo se pudo evaluar la cobertura en algunos subgrupos de interés; tampoco se pudo evaluar la oportunidad de vacunación, y si realmente ésta ocurrió lo más cercano posible al cumplimiento de los 12 y 18 meses del niño. Por la aplicación de los criterios de selección, excluimos información de un $20,0 \%$ de menores para la primera dosis y $30,0 \%$ para la de refuerzo, sin embargo esto fue necesario para evitar el sesgo de selección; la estimación global de la cobertura de vacunación debido al componente reporte de la madre y sus categorías de respuesta puede verse afectada por un sesgo de memoria o deseabilidad social; por último, no se pudo evaluar las contraindicaciones para la vacuna: inmunodeficiencia grave, reacción alérgica severa a una dosis previa de la vacuna y reacción a los componentes de la vacuna ${ }^{(8)}$

En conclusión, siete de cada diez niños peruanos de 12 a 59 meses de edad que participaron en la ENDES 2017 recibieron la primera dosis de la vacuna contra sarampión, y sólo cinco de diez recibieron la dosis de refuerzo. La cobertura de vacunación varió entre regiones, sin embargo, ninguna región del Perú llegó al 95,0\% de cobertura para la primera dosis y refuerzo. Entre los niños con controles de crecimiento y desarrollo en el sector público, la cobertura de la primera dosis llegó al $80,0 \%$, mientras que la de refuerzo fue $63,0 \%$. El principal factor modificable asociado a la vacunación contra sarampión fue el lugar donde se realizaron los controles de crecimiento y desarrollo, además, vivir cinco o más años en el lugar de residencia actual fue un factor independientemente asociado con la dosis de refuerzo.

Es fundamental para la eliminación del sarampión mantener altos niveles de inmunidad en la población, para ello es necesario alcanzar y mantener coberturas de al menos $95,0 \%$ tanto para la primera como para la segunda dosis. La ocurrencia de brotes de sarampión es uno de los primeros indicadores de debilidad del programa de vacunación en un país ${ }^{(3)}$. Recomendamos desarrollar investigaciones de implementación de políticas públicas, que evalúen la oportunidad de aplicación de esta vacuna, así como los factores que la determinan; de igual forma, complementar el abordaje del cumplimiento y cobertura del esquema de vacunación completo en niños a nivel nacional mediante estudios basados en encuestas poblaciones de salud. Por último, la implementación de mejoras en los registros rutinarios como el uso de registros nominales de vacunación electrónicos o cartillas electrónicas permitirá estimaciones de mayor validez de las coberturas de vacunación.

Contribuciones de autoría: La idea original de este estudio es de KVU, quien además fue responsable del análisis e interpretación de datos (con guía de JANO, FR y JCRH). KVU, JANO, FR y JCRH han participado del diseño del estudio, la redacción del artículo y han aprobado su versión final.

Fuentes de financiamiento: El presente estudio ha sido autofinanciado por KVU. La participación de JANO ha sido financiada por la Universidad Nacional de Cajamarca. La participación de JCRH y FR ha sido parcialmente financiada por el Instituto Nacional de Salud. Parte del tiempo de JCRH invertido en este proyecto fue también financiado por la Universidad de San Martín de Porres.

Conflictos de interés: FR se desempeña como Editor Científico de la Revista Peruana de Medicina Experimental y Salud Pública. Los demás autores declaran no tener conflictos de interés.

Material suplementario: Disponible en la versión electrónica de la RPMESP. 


\section{REFERENCIAS BIBLIOGRÁFICAS}

1. Demicheli V, Rivetti A, Debalini MG, Di Pietrantonj C. Vaccines for measles, mumps and rubella in children. Cochrane Database Syst Rev. 2012;(2):CD004407. doi: 10.1002/14651858.CD004407.pub3.

2. Thompson KM, Odahowski CL. Systematic Review of Health Economic Analyses of Measles and Rubella Immunization Interventions. Risk Anal Off Publ Soc Risk Anal. 2016;36(7):1297314. doi: 10.1111/risa.12331.

3. World Health Organization. Measles and Rubella Global Strategic Plan 2012-2020 [Internet]. Geneva: WHO; 2012 [citado el 24 de enero de 2019]. Disponible en: https://apps.who.int/iris/bitstream/handle/10665/44855/9789241503396_eng. pdf;jsessionid=2F9856D761A7234E9093CA3C0DCEEF66? sequence $=1$

4. Meissner HC, Strebel PM, Orenstein WA. Measles vaccines and the potential for worldwide eradication of measles. Pediatrics. 2004;114(4):1065-9. doi: 10.1542/peds.2004-0440.

5. Moss WJ, Strebel P. Biological Feasibility of Measles Eradication. J Infect Dis. 2011;204(Suppl 1):S47-53. doi: 10.1093/ infdis/jir065.

6. Anderson RM, May RM. Directly transmitted infections diseases: control by vaccination. Science. 1982;215(4536):1053-60. doi: $10.1126 /$ science.7063839.

7. Gay NJ. The theory of measles elimination: implications for the design of elimination strategies. J Infect Dis. 2004;189 Suppl 1:S27-35. doi: 10.1086/381592

8. Norma técnica de salud que establece el esquema nacional de vacunación. NTS N¹41-MINSA/2018/DGIESP. Resolución Ministerial N ${ }^{\circ} 719-2018 /$ MINSA (1 agosto 2018).Lima:MINSA;2018[Internet] [Citado el 12 de marzo 2019]. Disponible en: https://cdn.www.gob.pe/uploads/ document/file/300034/d177030_opt.PDF

9. Rengifo P. Situación epidemiológica de Sarampión- Rubéola, Perú (SE 01-SE 27,
2018). Boletín Epidemiológico del Perú [Internet]. 2018 [citado el 12 de marzo 2019];27(27):597-602. Disponible en: https://www.dge.gob.pe/portal/docs/ vigilancia/boletines/2018/27.pdf

10. Indicadores de la vigilancia conjunta de Sarampión - Rubéola. Boletín Epidemiológico del Perú [Internet]. 2018 [citado el 12 de marzo 2019] ;27(52):1291. Disponible en: https:// www.dge.gob.pe/portal/docs/ vigilancia/boletines/2018/52.pdf

11. Monitoring vaccination coverage: Defining the role of surveys. Vaccine. 2016;34(35):4103. doi: 10.1016/j. vaccine.2016.06.053.

12. Miles M, Ryman TK, Dietz V, Zell E, Luman ET. Validity of vaccination cards and parental recall to estimate vaccination coverage: a systematic review of the literature. Vaccine. 2013;31(12):15608. doi: 10.1016/j.vaccine.2012.10.089.

13. Roque Henriquez JC, Mera Villarreal JD, Romani Romani FR. Vacunación contra el virus de la hepatitis $B$ en recién nacidos de mujeres peruanas participantes de la Encuesta Demográfica y de Salud Familiar, 2016. An Fac Med. 2018;79(3):218-24. doi: 10.15381/anales.v79i3.15314.

14. ZaidiSMA, KhowajaS, Kumar Dharma V, Khan AJ, Chandir S. Coverage, timeliness, and determinants of immunization completion in Pakistan: Evidence from the Demographic and Health Survey (2006-07). Hum Vaccines Immunother. 2014;10(6):1712-20. doi: 10.4161/ hv.28621.b.

15. Mbengue MAS, Sarr M, Faye A, Badiane O, Camara FBN, Mboup S, et al. Determinants of complete immunization among senegalese children aged 12-23 months: evidence from the demographic and health survey. BMC Public Health. 2017;17(1):630. doi: 10.1186/s12889017-4493-3.

16. Ashbaugh HR, Hoff NA, Doshi RH, Alfonso VH, Gadoth A, Mukadi P, et al. Predictors of measles vaccination coverage among children 6-59 months of age in the Democratic Republic of the Congo. Vaccine. 2018;36(4):587-93. doi: 10.1016/j.vaccine.2017.11.049.

17. Lakew Y, Bekele A, Biadgilign S. Factors influencing fullimmunization coverage among 12-23 months of age children in Ethiopia: evidence from the national demographic and health survey in 2011. BMC Public Health. 2015;15:728. doi: 10.1186/s12889-015-2078-6.

18. Masters NB, Wagner AL, Carlson BF, Boulton ML. Vaccination timeliness and co-administration among Kenyan children. Vaccine. 2018; 36(11):1353-60. doi: 10.1016/j.vaccine.2018.02.001.

19. Organización Panamericana de la Salud. Organización Mundial de la Salud. Inmunización en las Américas, Resumen 2018 [Internet]. Ginebra: OPS, OMS; 2015. [citado el 29 de enero de 2019]. Disponible en: https://www. paho.org/hq/index.php?option $=$ com docman\&view=download\&category_ slug $=$ folleto $-2646 \&$ alias $=46874$ inmunizacion-en-las-americas-resumen2018\&Itemid $=270 \&$ lang $=$ es

20. Salam MA, Mboup A, Ly ID, Faye A, Niang FB, Thiam M, et al. Vaccination coverage and immunization timeliness among children aged 12-23 months in Senegal: a Kaplan-Meier and Cox regression analysis approach. Pan Afr Med J. 2017;27(Suppl 3):8. doi: 10.11604/ pamj.supp.2017.27.3.11534.

Correspondencia: Joel Christian Roque Henriquez

Dirección: Cápac Yupanqui 1400, Jesús María. Lima, Perú

Teléfono: (051) 940430215

Correo electrónico:jroque@ins.gob.pe 